Anaesthesist 2010 · 59:593-594

DOI 10.1007/s00101-010-1746-2

Online publiziert: 14. Juli 2010

(c) Springer-Verlag 2010

\author{
M. Gama de Abreu ${ }^{1,2}$ • A. Güldner ${ }^{2} \cdot$ T. Koch ${ }^{1}$ \\ ${ }^{1}$ Klinik für Anästhesie und Intensivtherapie, \\ Universitätsklinikum Carl Gustav Carus, Technische Universität Dresden \\ 2 Pulmonary Engineering Group, Universitätsklinikum Carl Gustav Carus, \\ Technische Universität Dresden
}

\section{Protektive Beatmung im Operationssaal}

\section{Fehlende klinische Evidenz ist nicht mit einer Evidenz fehlender Wirkung gleichzusetzen}

Die erste Beschreibung des akuten Lungenversagens (,acute respiratory distress syndrome“, ARDS) und des respiratorischen Managements dieses Syndroms ist auf das Jahr 1967 durch Ashbaugh et al. [1] zurückzuführen. Leitsymptome und -signale dieses Syndroms waren Tachypnoe, Hypoxämie und reduzierte Dehnbarkeit des respiratorischen Systems, die nicht nur nach primärer Lungenschädigung, wie z. B. bei Pneumonie oder Lungenkontusion, sondern auch nach sekundärer Lungenschädigung, d. h. nicht nach unmittelbar von den Lungen ausgehenden Erkrankungen wie z. B. Pankreatitis, zu verzeichnen waren.

Obwohl Veränderungen des Lungenparenchyms mit u. a. hyalinen Membranen, Ödem und Bildung von Atelektasen in dieser Publikation bereits erkannt wurden, stand die Therapie der refraktären Hypoxämie im Vordergrund [1]. Dementsprechend fand die Verwendung von höheren Tidalvolumina und inspiratorischen Sauerstoffkonzentrationen $\left(\mathrm{F}_{\mathrm{I}} \mathrm{O}_{2}\right)$ mit moderatem positiven end-exspiratorischen Druck („positive end-expiratory pressure“, PEEP) statt. Zirka 4 Jahre später wurde durch die gleiche Gruppe deutlich gemacht, dass die Priorität der Therapie des ARDS in der Hypoxämie festgelegt war [13], was häufig mit einer aggressiven Beatmungsstrategie einherging.

In der Zeit zwischen diesen Publikationen wurde eine theoretische und physika- lische Analyse der Kräfte, die sich auf eine inhomogene Lungenstruktur auswirken, veröffentlicht [11]. Es wurde postuliert, dass die Schnittstelle zwischen benachbarten Lungenbezirken, die unterschiedliche mechanische Eigenschaften aufweisen, erhöhten mechanischen Stress erleiden. Diese Ergebnisse fanden nicht die notwendige Beachtung, und die Anwendung von Tidalvolumina von $12-15 \mathrm{ml} /$ kgKG im Rahmen der maschinellen Beatmung des ARDS-Patienten setzte sich noch einige Jahre fort, selbst als die möglichen schädlichen Auswirkungen der Beatmung auf das Lungenparenchym schon erkannt waren und das Konzept der „baby lung“, d. h. einer reduzierten Lungenfläche mit beibehaltener „compliance“, von Gattinoni et al. [7] vorgeschlagen wurde. In einer retrospektiven Studie hat Hickling [9] kurz danach festgestellt, dass die Anwendung niedrigerer Beatmungsspitzendrücke bei Patienten mit ARDS mit einer reduzierten Letalitätsrate verbunden war.

Es wurde jedoch früh erkannt, dass die Schädigung des Lungenparenchyms nicht nur durch direkte mechanische Ruptur des Gewebes entsteht, sondern auch durch die Verwandlung des mechanischen Stresses in eine biochemische Reaktion mit Freisetzung von Zytokinen und sogar Dekompartmentierung des inflammatorischen Vorgangs $[8,15,20]$. In kleinen Tieren kann die Beatmung mit exzessiv hohen Tidalvolumina von $30-40 \mathrm{ml} / \mathrm{kg}$ KG sogar das ARDS hervorrufen [6], dies ist der sog. beatmungsinduzierte Lungenschaden („ventilator induced lung injury“, VILI). An Patienten geht man eher davon aus, dass VILI keine Rolle spielt und dass die Beatmungsstrategie erst die Lungen schädigen kann, wenn diese sich bereits in einem inflammatorischen Prozess befinden. Dabei ist vielmehr die Rede von einem beatmungsassoziierten Lungenschaden („ventilator associated lung injury“, VALI), was mit dem Konzept eines notwendigen primären Reizes oder der „Double-hit“-Theorie in Verbindung steht [21]. Folgt man diesem - nicht unumstrittenen $[5,17]$ - Konzept, würden nur vorher geschädigte Lungen von einer protektiven Beatmung mit niedrigeren Tidalvolumina profitieren [14]. Umgekehrt wird befürchtet, dass niedrigere Tidalvolumina und Beatmungsdrücke bei gesunden Lungen Nachteile im Sinne der Entwicklung eines vermehrten Kollapses abhängiger Abschnitte nach sich ziehen können, wobei auch diese Auffassung nicht unwidersprochen geblieben ist [3].

In dieser Ausgabe von Der Anaesthesist knüpfen David, Bodenstein und Markstaller an die aktuelle Diskussion an und setzen sich detailliert mit dem Thema der protektiven Beatmung im OP auseinander [4]. Dabei ist der Hauptschlussfolgerung der Autoren zuzustimmen, wonach nämlich die heutige Datenlage die Über- 
tragung des Protektiven-Beatmung-Konzeptes auf die gesunden Lungen nicht unterstützt. Die Frage nach optimalen Beatmungsstrategien bei lungengesunden $\mathrm{Pa}$ tienten ist bislang nicht befriedigend beantwortet. Dennoch ist hier Vorsicht geboten. Auch wenn die klinische Evidenz fehlt, sollte sich der Anästhesist fragen: Was ist die Rationale für die Anwendung einer nichtprotektiven Beatmung bei lungengesunden Patienten? Die befürchtete Bildung von Atelektasen während der Beatmung mit niedrigeren Tidalvolumina kann durch die Anwendung adäquater PEEP-Werte $z$. T. bis fast ganz vermieden werden. Ferner sind niedrigere Tidalvolumina von $6 \mathrm{ml} / \mathrm{kgKG}$, wie es bei der Beatmung des ARDS empfohlen wird [19], nicht wirklich niedrig. Säugetiere, ob Maus, Mensch oder Wal, weisen ein durchschnittliches Tidalvolumen von ca. $6 \mathrm{ml} / \mathrm{kgKG}$ auf [18]. Außerdem stellt sich die Frage, ob ein ausgedehnter chirurgischer Eingriff mit Freisetzung von inflammatorischen Mediatoren in die Blutbahn $[12,16]$ nicht bereits als primärer „hit“ der Lungen anzusehen ist.

Die Begrenzung von Tidalvolumina auf $6 \mathrm{ml} / \mathrm{kgKG}$ (oder sogar niedriger im Rahmen der Einlungenanästhesie) und inspiratorischem Plateaudruck auf $20 \mathrm{~cm}$ $\mathrm{H}_{2} \mathrm{O}$, wenn die Compliance des respiratorischen Systems nicht eingeschränkt ist (z. B. bei laparoskopischen Eingriffen, erhöhtem abdominalen Druck und Thoraxverbrennung), erscheint bei ausgedehnten chirurgischen Eingriffen sinnvoll, bis klinische Studien dies endgültig geklärt haben. Wenn die chirurgische Intervention jedoch mit einer minimalen Freisetzung von inflammatorischen Mediatoren verbunden ist, ist die Relevanz der protektiven Beatmung anzuzweifeln bzw. sogar infrage zu stellen. In einer ganz aktuellen experimentellen Arbeit haben Hong et al. [10] nämlich gezeigt, dass bei gesunden Lungen und minimaler chirurgischer Intervention die Beatmung mit Tidalvolumina von $15 \mathrm{ml} / \mathrm{kgKG}$ und einem PEEP von $3 \mathrm{cmH}_{2} \mathrm{O} \mathrm{zu}$ weniger Inflammation und Lungenschädigung führt als eine $\mathrm{Be}$ atmung mit Tidalvolumina von $6 \mathrm{ml} / \mathrm{kg}$ $\mathrm{KG}$ und einem PEEP von 3 bzw. $10 \mathrm{~cm}-$ $\mathrm{H}_{2} \mathrm{O}$. Inwiefern sich diese experimentellen Ergebnisse reproduzieren bzw. auf die kli- nische Praxis übertragen lassen, ist noch ungewiss.

Die Frage nach dem adäquaten PEEP bleibt im Kontext der protektiven Beatmung im OP bislang ebenso unbeantwortet, wie es beim ARDS der Fall ist. Intraoperativ muss noch berücksichtigt werden, dass die Erhöhung des PEEP und somit des mittleren Atemwegsdrucks mit einem Anstieg des Zentralvenendrucks einhergehen kann, der unter bestimmten chirurgischen Bedingungen Nachteile mit sich bringen kann, wie z. B. in der Leberchirurgie. Außerdem ist es bekannt, dass sich die Kombination von niedrigeren Tidalvolumina mit inadäquaten hohen PEEP-Werten wenig protektiv bis lungenschädlich auswirken kann [2].

Unter Berücksichtigung von potenziellen Nebenwirkungen ist die Meinung vertretbar, dass die Prinzipien der protektiven Beatmung mit niedrigeren Tidalvolumina bei ausgedehnten chirurgischen Eingriffen bzw. erhöhtem Risiko, ein akutes Lungenversagen $\mathrm{zu}$ entwickeln, bis zum Beweis des Gegenteils auch im OP Anwendung finden sollen. Die pathophysiologische Rationale ist evident, und die heute fehlende klinische Evidenz ist nicht mit einer Evidenz fehlender Wirkung gleichzusetzen.

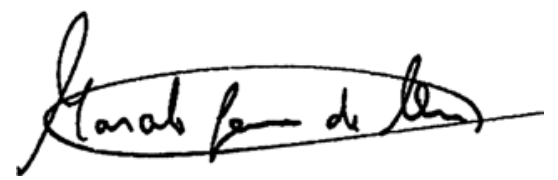

M. Gama de Abreu

\section{Korrespondenzadresse}

\section{Prof. Dr. M. Gama de Abreu}

Klinik für Anästhesie und Intensivtherapie, Universitätsklinikum Carl Gustav Carus, Technische Universität Dresden Fetscherstraße 74, 01307 Dresden mgabreu@aol.com

\section{Literatur}

1. Ashbaugh DG, Boyd Bigelow D, Petty Tl et al. (1967) Acute respiratory distress in adults. Lancet 2:319-323

2. Barbas CS, de Matos GF, Pincelli MP et al. (2005) Mechanical ventilation in acute respiratory failure: recruitment and high positive end-expiratory pressure are necessary. Curr Opin Crit Care 11:18-28

3. Cai $\mathrm{H}$, Gong $\mathrm{H}$, Zhang L et al. (2007) Effect of low tidal volume ventilation on atelectasis in patients during general anesthesia: a computed tomographic scan. J Clin Anesth 19:125-129
4. David M, Bodenstein M, Markstaller K (2010) Protektive Beatmungstherapie: Auch für den OP relevant? Anaesthesist, doi 10.1007/s00101-010-1743-5

5. Determann RM, Royakkers A, Wolthuis EK et al. (2010) Ventilation with lower tidal volumes as compared with conventional tidal volumes for patients without acute lung injury: a preventive randomized controlled trial. Crit Care 14:R1

6. Dreyfuss D, Saumon G (1998) Ventilator-induced lung injury. Am J Respir Crit Care Med 157:294323

7. Gattinoni L, Pesenti A, Avalli L et al. (1987) Pressure-volume curve of total respiratory system in acute respiratory failure. Computed tomographic scan study. Am Rev Respir Dis 136:730-736

8. Held HD, Boettcher S, Hamman L et al. (2001) Ventilation-induced chemokine and cytokine release is associated with activation of nuclear factor-kap$\mathrm{paB}$ and is blocked by steroids. Am J Respir Crit Care Med 163:711-716

9. Hickling KG (1990) Ventilatory management of ARDS: can it affect the outcome? Intensive Care Med 16:219-226

10. Hong Cm, Xu Dz, Lu Q et al. (2010) Low tidal volume and high positive end-expiratory pressure mechanical ventilation results in increased inflammation and ventilator-associated lung injury in normal lungs. Anesth Analg 110:1652-1660

11. Mead J, Takishima T, Leith D (1970) Stress distribution in lungs: a model of pulmonary elasticity. J Appl Physiol 28:596-608

12. Menger MD, Vollmar B (2004) Surgical trauma: hyperinflammation versus immunosuppression? Langenbecks Arch Surg 389:475-484

13. Petty TI, Ashbaugh DG (1971) The adult respiratory distress syndrome - clinical features, factors influencing prognosis and principles of management. Chest 60:233-239

14. Putensen C, Wrigge $H$ (2007) Tidal volumes in patients with normal lungs: one for all or the less, the better? Anesthesiology 106:1085

15. Ranieri VM, Suter PM, Tortorella C (1999) Effect of mechanical ventilation on inflammatory mediators in patients with acute respiratory distress syndrome: a randomized controlled trial. JAMA 282:54-61

16. Roumen RM, Hendriks T, van der Ven-Jongekrijg J et al. (1993) Cytokine patterns in patients after major vascular surgery, hemorrhagic shock, and severe blunt trauma. Relation with subsequent adult respiratory distress syndrome and multiple organ failure. Ann Surg 218:769-776

17. Schultz MJ (2008) Lung-protective mechanical ventilation with lower tidal volumes in patients not suffering from acute lung injury: a review of clinical studies. Med Sci Monit 14:RA22-26

18. Tenney SM, Remmers JE (1963) Comparative quantitative morphology of the mammalian lung: diffusing area. Nature 197:54

19. The Acute Respiratory Distress Syndrome Network (2000) Ventilation with lower tidal volumes as compared with traditional tidal volumes for acute lung injury and the acute respiratory distress syndrome. N Engl J Med 342:1301-1308

20. Tremblay L, Valenza F, Ribeiro SP et al. (1997) Injurious ventilatory strategies increase cytokines and c-fos expression in an isolated rat lung. J Clin Invest 99:944-952

21. Whitehead TC, Zhang H, Mullen B et al. (2004) Effect of mechanical ventilation on cytokine response to intratracheal lipopolysaccharide. Anesthesiology 101:52-58 